\title{
Digital Quantum Simulation of Spin Models with Circuit Quantum Electrodynamics
}

\author{
Y. Salathé,,${ }^{1, *}$ M. Mondal, ${ }^{1}$ M. Oppliger, ${ }^{1}$ J. Heinsoo, ${ }^{1}$ P. Kurpiers,,${ }^{1}$ A. Potočnik, ${ }^{1}$ A. Mezzacapo, ${ }^{2}$ \\ U. Las Heras, ${ }^{2}$ L. Lamata, ${ }^{2}$ E. Solano, ${ }^{2,3}$ S. Filipp, ${ }^{1, \dagger}$ and A. Wallraff ${ }^{1}$ \\ ${ }^{1}$ Department of Physics, ETH Zürich, CH-8093 Zürich, Switzerland \\ ${ }^{2}$ Department of Physical Chemistry, University of the Basque Country UPV/EHU, \\ Apartado 644, E-48080 Bilbao, Spain \\ ${ }^{3}$ IKERBASQUE, Basque Foundation for Science, Maria Diaz de Haro 3, 48013 Bilbao, Spain
}

(Received 6 March 2015; published 17 June 2015)

\begin{abstract}
Systems of interacting quantum spins show a rich spectrum of quantum phases and display interesting many-body dynamics. Computing characteristics of even small systems on conventional computers poses significant challenges. A quantum simulator has the potential to outperform standard computers in calculating the evolution of complex quantum systems. Here, we perform a digital quantum simulation of the paradigmatic Heisenberg and Ising interacting spin models using a two transmon-qubit circuit quantum electrodynamics setup. We make use of the exchange interaction naturally present in the simulator to construct a digital decomposition of the model-specific evolution and extract its full dynamics. This approach is universal and efficient, employing only resources that are polynomial in the number of spins, and indicates a path towards the controlled simulation of general spin dynamics in superconducting qubit platforms.
\end{abstract}

DOI: 10.1103/PhysRevX.5.021027

Quantum simulations using well-controllable quantum systems to simulate the properties of another less tractable one $[1,2]$ are expected to be able to predict the properties and dynamics of diverse systems in condensed matter $[3,4]$, quantum chemistry [5], and high-energy physics [6,7]. In particular, quantum simulations are expected to provide new insights into open problems, such as modeling high- $T_{c}$ superconductivity [8], thermalization [9], and nonequilibrium dynamics [10]. Up to now, several prototypical quantum simulations have been proposed and realized in trapped ions [11], cold atoms [12], and quantum photonics [13]. These systems have fundamentally different intrinsic properties offering complementary paths for realizing quantum simulators to which circuit QED platforms are expected to contribute. Examples of simulations carried out include spin models [14-16], many-body physics [17], and relativistic quantum mechanics [18]. In the field of superconducting circuits, quantum simulations are still in their infancy [19]. Topological properties [20,21] have been simulated recently, as have been fermionic models [22].

\footnotetext{
*ysalathe@phys.ethz.ch

Now at: IBM T. J. Watson Research Center, Yorktown Heights, NY 10598, USA.

Published by the American Physical Society under the terms of the Creative Commons Attribution 3.0 License. Further distribution of this work must maintain attribution to the author $(s)$ and the published article's title, journal citation, and DOI.
}

\author{
Subject Areas: Condensed Matter Physics, \\ Quantum Information, \\ Superconductivity
}

Quantum simulators are typically classified into two main categories, namely, analog and digital. Analog quantum simulators are designed to display intrinsic dynamics that are equivalent to those of the simulated system. While this approach is not universal, it features control of the relevant Hamiltonian parameters better than in the system to be simulated. Instead, digital quantum simulators [2] can reproduce the dynamics of a quantum system via a universal digital decomposition of its Hamiltonian $H=$ $\sum_{k} H_{k}$ into efficient elementary gates realizing $H_{k}$. This approach is based on the Suzuki-Lie-Trotter expansion of the time evolution $U(t)=e^{-i H t}=\lim _{n \rightarrow \infty}\left(\prod_{k=1}^{N} e^{-i H_{k} t / n}\right)^{n}$ and was recently demonstrated experimentally in a trappedion digital quantum simulator [15]. Variants of this digital protocol make use of fractal approximations [23], adaptive time steps for time-dependent Hamiltonians [24], and heralded protocols for the implementation of linear combinations of operators [25].

Here, we demonstrate digital quantum simulation of spin systems [16] in an architecture known as circuit QED [26]. Our experiments are carried out with two superconducting transmon qubits [27] coupled dispersively to a common mode of a coplanar waveguide resonator (see Appendix A for the device layout and setup diagram). We operate the circuit at $30 \mathrm{mK}$ in a dilution refrigerator. The qubits Q1 and Q2 interact with a coplanar waveguide resonator with a fundamental resonance frequency at $7.14 \mathrm{GHz}$, which serves both as a quantum bus [28] and for readout [29]. 
The natural two-qubit interaction is the $X Y$ exchange coupling [28] $H_{1,2}^{x y}=(J / 2)\left(\sigma_{1}^{x} \sigma_{2}^{x}+\sigma_{1}^{y} \sigma_{2}^{y}\right)$ mediated by virtual photons in a common cavity mode, which we also refer to as the $X Y$ interaction. Here, $\sigma_{i}^{x, y}$ are the Pauli operators acting on qubit $i$, and $J$ denotes the effective qubit-qubit coupling strength [30]. The $X Y$ interaction is activated by tuning the transition frequency of qubit Q1 $(5.44 \mathrm{GHz})$ into resonance with qubit Q2 $(5.24 \mathrm{GHz})$ for a time $\tau$ using nanosecond time scale magnetic flux bias pulses [31] (see Appendix B). When the qubit transition frequencies are degenerate, the resonator-mediated coupling strength is spectroscopically determined to be $J=-40.4 \mathrm{MHz}$. To make the presentation of the simulation results independent of the actual $J$, we express the interaction time $\tau$ for a given $J$ in terms of the acquired quantum phase angle $2|J| \tau$. In our setup, the action of the $X Y$ gate [Fig. 1(a)] is characterized by full process tomography for a complete set of 16 initial two-qubit states and a series of 25 different interaction times $\tau$ finding process fidelities no lower than 89\% (see Appendix D).

(a)
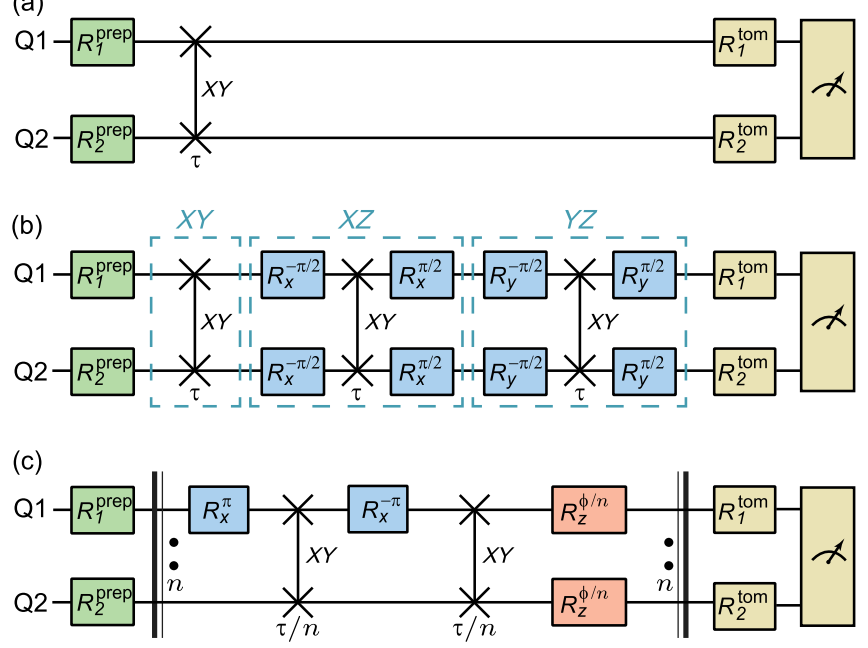

FIG. 1. (a) Circuit diagram to characterize the $X Y$ exchange interaction on qubits Q1 and Q2 symbolized by the vertical line $(\times)$, which is activated for a time $\tau$. To perform standard process tomography of this interaction, separable initial states are prepared using single-qubit rotations $R_{1,2}^{\text {prep }}$ (green) in the beginning and the final state is characterized using single-qubit basis rotations $R_{1,2}^{\text {tom }}$ and joint two-qubit readout (yellow). (b) Digital quantum simulation of the two-spin Heisenberg $(X Y Z)$ interaction for time $\tau$. The first step after state preparation is to apply the $X Y$ gate for a time $\tau$ (dashed box labeled as $X Y$ ). In the second and third steps (dashed boxes with labels $X Z$ and $Y Z$ ), $X Z$ and $Y Z$ gates are realized using single-qubit rotations $R_{x, y}^{ \pm \pi / 2}$ (blue) by an angle $\pm \pi / 2$ about the $x$ or $y$ axis transforming the basis in which the $X Y$ gate acts. (c) Protocol to decompose and simulate Ising spin dynamics in a homogeneous transverse magnetic field. The circuit between the bold vertical bars with two dots is repeated $n$ times, invoking each $X Y$ and phase gates for a time $\tau / n$. See text for details. The actual pulse scheme is provided in Appendix C.
In Figs. 2(a) and 2(b), we present nonstationary spin dynamics under the $X Y$ exchange interaction for a characteristic initial two-qubit state $|\uparrow\rangle(|\uparrow\rangle+|\downarrow\rangle) / \sqrt{2}$ with spins pointing in perpendicular directions along $+\mathbf{z}$ and $+\mathbf{x}$, respectively. During the $X Y$ interaction, the state of one spin is gradually swapped to the other spin and vice versa, with a phase angle of $\pi / 2$. This corresponds to the iSWAP gate [32]. As a consequence, the measured Bloch vectors move along the $Y Z$ and $X Z$ planes. For a quantum phase angle of $2|J| \tau=\pi$, they point along the $+\mathbf{y}$ and $+\mathbf{z}$ directions, respectively, in good agreement with the ideal unitary time evolution indicated by dashed lines in Figs. 2(a) and 2(b). We also find that the two-qubit entanglement characterized by the measured negativity [33] of 0.246 is close to the maximum expected value of 0.25 for this initial state at a quantum phase angle of $\pi / 2$. As a consequence, the Bloch vectors do not remain on the surface of the Bloch sphere but rather lie within the sphere.

The anisotropic Heisenberg model describes spins interacting in three spatial dimensions,

$$
H_{x y z}=\sum_{(i, j)}\left(J_{x} \sigma_{i}^{x} \sigma_{j}^{x}+J_{y} \sigma_{i}^{y} \sigma_{j}^{y}+J_{z} \sigma_{i}^{z} \sigma_{j}^{z}\right)
$$

where the sum is taken over pairs of neighboring spins $i$ and $j . J_{x}, J_{y}$, and $J_{z}$ are the couplings of the spins along the $x, y$, and $z$ coordinates, respectively. Since it does not occur naturally in circuit QED, we decompose the Heisenberg interaction into a sequence of $X Y$ and single-qubit gates, as shown in Fig. 1(b). We combine three successive effective $X Y, X Z$, and $Y Z$ gates derived from the $X Y$ gate by basis transformations [16] to realize the isotropic Heisenberg model with $J_{x}=J_{y}=J_{z}=J$ versus interaction time $\tau$. Since the $X Y, X Z$, and $Y Z$ operators commute for two spins, the Trotter formula is exact after a single step.

To compare the Heisenberg $(X Y Z)$ interaction with the $X Y$ exchange interaction, we prepare the same initial state as presented in Figs. 2(a) and 2(b). The isotropic Heisenberg interaction described by the scalar product between two vectorial spin-1/2 operators preserves the angle between the two spins. As a result, the initially perpendicular Bloch vectors of qubits Q1 and Q2 remain perpendicular during the interaction [Fig. 2(c)] and rotate clockwise along an elliptical path that spans a plane perpendicular to the diagonal at half-angle between the two Bloch vectors [Fig. 2(c)].

In accordance with theory, the $X Y Z$ interaction leads to a full SWAP operation for a quantum phase angle of $2|J| \tau=\pi / 2$, where the Bloch vectors point along the $+\mathbf{x}$ and $+\mathbf{z}$ directions. For the given initial state, we observe a maximum negativity of 0.210 close to the expected value of 0.25 for the Heisenberg interaction at a quantum phase angle of $2|J| \tau=\pi / 4$. As for the $X Y$ interaction, we characterize the Heisenberg interaction with 
(a)

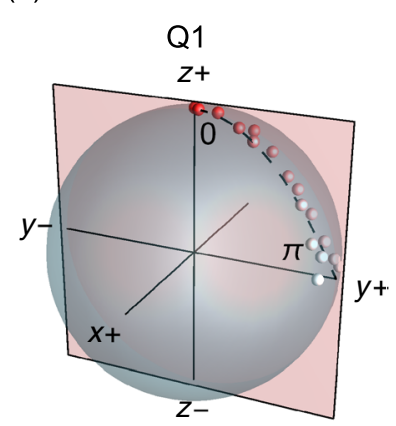

$X Y$

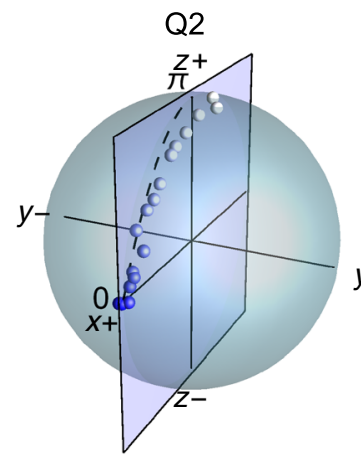

(c)

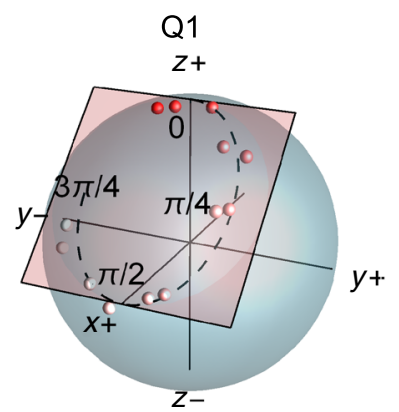

$X Y Z$

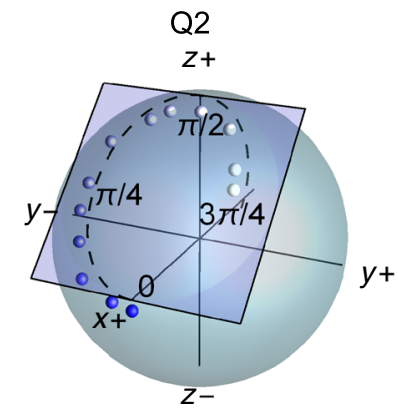

(b)

$\left\langle\sigma_{1}^{x}\right\rangle,\left\langle\sigma_{2}^{x}\right\rangle \quad\left\langle\sigma_{1}^{y}\right\rangle,\left\langle\sigma_{2}^{y}\right\rangle$ $\left\langle\sigma_{1}^{z}\right\rangle,\left\langle\sigma_{2}^{z}\right\rangle$

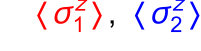

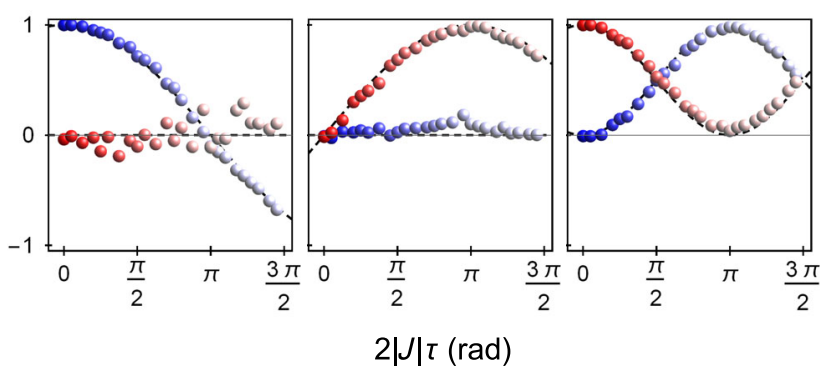

(d)

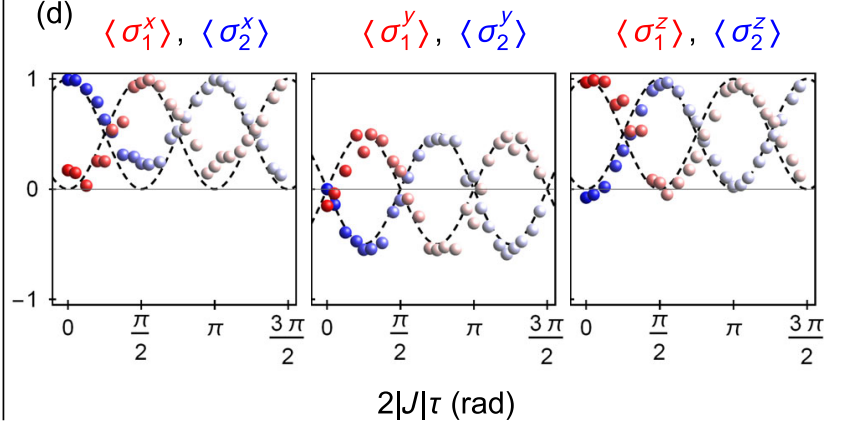

FIG. 2. (a) Experimentally determined coordinates of the Bloch vectors during exchange $(X Y)$ interaction represented by small red $(\mathrm{Q} 1)$ and blue $(\mathrm{Q} 2)$ points are compared to the ideal paths shown as dashed lines in the $X Y$ model. The ideal paths are in the $Y Z$ and $X Z$ planes shown as blue and red planes intersecting the Bloch sphere. The time evolution is indicated by the saturation of the colors as the quantum phase angle $2|J| \tau$ advances from 0 (saturated) to $\pi$ (unsaturated). (b) Measured expectation values of the Pauli operators $\sigma_{1,2}^{x, y, z}$ for qubits Q1 (red points) and Q2 (blue points), respectively, for the $X Y$ interaction as a function of the quantum phase angle $2|J| \tau$ along with the ideal evolution (dashed line). (c) Evolution of the Bloch vector for the quantum simulation of the isotropic Heisenberg interaction versus quantum phase angles from 0 to $3 \pi / 4$. The path of the Bloch vectors of qubits Q1 and Q2 spans the plane indicated by the rectangular sheets intersecting the Bloch spheres. (d) As in (b) for the Heisenberg interaction.

standard process tomography, finding fidelities above $82 \%$ for all quantum phase angles $2|J| \tau$.

Next, we consider the quantum simulation of the Ising model with a transverse homogeneous magnetic field,

$$
H_{I}=J \sum_{(i, j)} \sigma_{i}^{x} \sigma_{j}^{x}+\frac{B}{2} \sum_{i} \sigma_{i}^{z},
$$

where the magnetic field $B$ pointing along the $z$ axis is perpendicular to the interaction given by $J \sigma_{i}^{x} \sigma_{j}^{x}$. Since the two-spin evolution [Fig. 1(c)] is decomposed into twoqubit $X Y$ and single-qubit $Z$ gates which do not commute, the transverse-field Ising dynamics is only recovered using the Trotter expansion in the limit of a large number of steps $n$ for an interaction time of $\tau / n$ in each step. To realize the Ising interaction term using the exchange interaction, the $X Y$ gate is applied twice for a time $\tau / n$, once enclosed by a pair of $\pi$ pulses on qubit Q1. This leads to a change of sign of the $\sigma_{1}^{y} \sigma_{2}^{y}$ term, which thus gets canceled when added to the bare $X Y$ gate. The external magnetic field part of the Hamiltonian is realized as single-qubit phase gates $R_{z}^{\phi}$, which rotate the Bloch vector about the $z$ axis by an angle
$\phi=B \tau / n$ per Trotter step. These gates are realized by detuning the respective qubit by an amount $\delta$ from its idle frequency corresponding to an effective $B$-field strength of $B=2 \pi \delta$.

We experimentally simulate the nonstationary dynamics of two spins in this model for the initial state $|\uparrow\rangle(|\uparrow\rangle-i|\downarrow\rangle) / \sqrt{2}$, which is well suited to assess the simulation performance. In Fig. 3(a), expectation values for the digital simulation of the $\sigma_{1,2}^{z}$ components of the two spins are shown, as well as the two-point correlation function $\left\langle\sigma_{1}^{x} \sigma_{2}^{x}\right\rangle$. The $\sigma_{1,2}^{z}$ components of the spins represented by the red and blue data sets in Fig. 3(a), respectively, oscillate with a dominant frequency component of $2 J$ due to the presence of the interaction term $\propto \sigma_{1}^{x} \sigma_{2}^{x}$. Likewise, the $X X$ correlation $\left\langle\sigma_{1}^{x} \sigma_{2}^{x}\right\rangle$ represented by the yellow data set in Fig. 3(a) is nonstationary and oscillates at rate $2 \sqrt{B^{2}+J^{2}}=2 \sqrt{10} J \approx 6.3 J$ due to the presence of a magnetic field of strength $B=3 J$. The evolution of the measured final state shows agreement with a theoretical model [solid lines in Fig. 3(a)], which takes into account dissipation and decoherence with deviations being dominated by systematic gate errors (see Appendix E). 
(a) No. of Trotter steps: 1
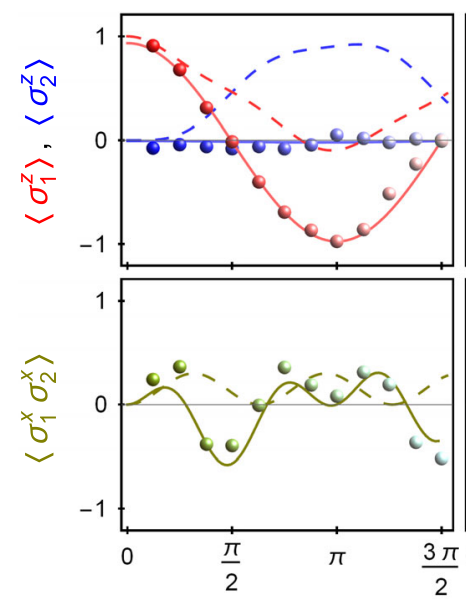

2
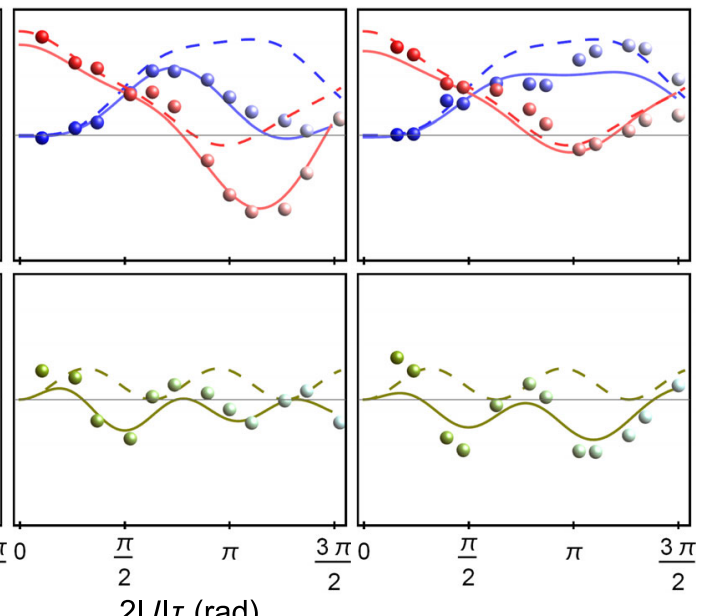

$2|J| \tau(\mathrm{rad})$ (b)

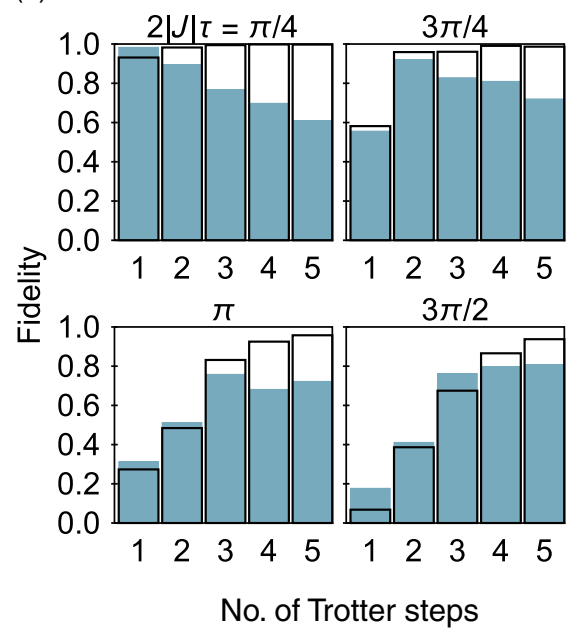

FIG. 3. (a) Digital quantum simulation of the Ising model with transverse homogeneous magnetic field using 1-3 Trotter steps. Shown are the $z$ components $\left\langle\sigma_{1}^{z}\right\rangle$ of qubit Q1 (red) and $\left\langle\sigma_{2}^{z}\right\rangle$ of qubit Q2 (blue) and the two-point correlation function in the $x$ direction $\left\langle\sigma_{1}^{x} \sigma_{2}^{x}\right\rangle$ (yellow points) of the spins as a function of the quantum phase angle $2|J| \tau$ for the initial state $|\uparrow\rangle(|\uparrow\rangle-i|\downarrow\rangle) / \sqrt{2}$ and a magnetic field strength $B=3 J$. Theoretically expected results take systematic phase offsets and finite coherence of the qubits into account (solid curves). The ideal dynamics are obtained from the time-dependent Schrödinger equation for the Ising Hamiltonian (dashed lines). (b) Fidelity with respect to the exactly solved Ising model for displayed quantum phase angles of the final state after ideal unitary evolution in the simulation protocol for $n$ Trotter steps (wire frames) and experimentally obtained final state (colored bars).

In Fig. 3(b), the fidelity of the simulated state is compared to the expected state at characteristic quantum phase angles for both the experimental realization (colored bars) and the ideal Trotter approximation (wire frames) after the $n$th step. In an ideal digital quantum simulator, the theoretical fidelity (wire frame) converges for an increasing number of steps $n$ [Fig. 3(b)]. The experimental fidelity, however, reaches a maximum for a finite number of steps [Fig. 3(b)], after which it starts to decrease due to gate errors and decoherence [16]. As expected, the Trotter approximation converges faster for smaller quantum phase angles $2|J| \tau$. For $2|J| \tau=\pi / 4$, the peak experimental fidelity [Fig. 3(b)] of $98.3 \%$ is already observed for $n=1$, whereas for $2|J| \tau=3 \pi / 2$, the optimum of $80.7 \%$ is observed for $n=5$.

To simulate large lattices, it is important to notice that the quantum resources needed to realize one digital step scale polynomially in the size of the problem, while the total number of gates for the whole protocol scales subpolynomially in the digital error [34]. The required reduction in the gate errors is expected to be achievable by incorporating optimal control techniques for pulse shaping [35] and by optimizing the fabrication process to improve the coherence times. Furthermore, techniques to perform multiplexed readout [36] and control of spectrally close qubits [37] will improve scalability. In addition, the usage of cryogenic switches or even cryogenic control logic circuits [38] is expected to reduce the required resources in terms of both the number of signal sources and the number of microwave lines affecting the heat load of the cryostat.
In future experiments, transmission line resonators may provide a means to design multiqubit devices with nonlocal qubit-qubit couplings that directly reflect the lattice topology of spin systems such as frustrated magnets. This design flexibility, in combination with fast gate operations [39], fast initialization [40,41], and high-fidelity readout based on quantum-limited amplifiers [42,43], sets the circuit QED platform apart from other quantum simulators for investigating static $[14,44,45]$ or dynamic $[15,46-50]$ properties of systems with Ising and Heisenberg types of spin-spin interaction. Moreover, the incorporation of cavity modes as explicit degrees of freedom in the simulated models [51], following an analog-digital approach, will be instrumental to scale the system to larger Hilbert-space dimensions. First steps in these directions have been taken by realizing and working with devices combining multiple qubits with multiple resonators [52-55]. With this, the circuit QED architecture offers considerable potential for surpassing the limitations of classical simulations, which can be facilitated by using efficient digital decompositions of spin Hamiltonians as pursued in this work.

The authors would like to thank Abdufarrukh Abdumalikov and Marek Pechal for helpful discussions. Furthermore, we owe gratitude to Lars Steffen, Arkady Fedorov, Christopher Eichler, Mathias Baur, and Jonas Mlynek who contributed to our experimental setup. We would also like to thank Tim Menke and Andreas Landig for contributions to the calibration software used in the present experiment. We acknowledge financial support from Eidgenössische Technische Hochschule Zurich (ETH 
Zurich), the Swiss National Science Foundation National Centre of Competence in Research "Quantum Science \& Technology," the Basque Government IT472-10, Spanish MINECO FIS2012-36673-C03-02, Ramón y Cajal Grant No. RYC-2012-11391, UPV/EHU Project No. EHUA14/04, UPV/EHU UFI 11/55, and a UPV/EHU PhD grant, and PROMISCE and SCALEQIT European projects.

\section{APPENDIX A: CHIP ARCHITECTURE AND MEASUREMENT SETUP}

The present experiment is performed using two superconducting transmon [27] qubits Q1 and Q2 and one coplanar waveguide resonator R1 on a microchip (Fig. 4). The resonator R1 has a fundamental resonance frequency of $\nu_{r}=7.14 \mathrm{GHz}$. From spectroscopic measurements, we determine the maximum transition frequencies $\nu_{\max }=\{5.55,5.53\} \mathrm{GHz}$ and charging energies $E_{C} / h \approx$ $\{260,260\} \mathrm{MHz}$ of qubits Q1 and Q2, respectively, where $h$ is the Planck constant. Qubits Q1 and Q2 are coupled to resonator $\mathrm{R} 1$ with coupling strengths $g / 2 \pi \approx\{120,120\} \mathrm{MHz}$. For this experiment, the qubit transition frequencies in their idle state are offset to $\nu=$ $\{5.440,5.240\} \mathrm{GHz}$ by applying a constant magnetic flux threading their SQUID loops with miniature superconducting coils mounted underneath the chip. At these idle frequencies, the measured energy relaxation and coherence times are $T_{1}=\{7.1,6.7\} \mu \mathrm{s}$ and $T_{2}=\{5.4,4.9\} \mu \mathrm{s}$, respectively. The transition frequencies of qubits Q3 and Q4 are tuned to 4.5 and $6.1 \mathrm{GHz}$ such that they do not interact with Q1 and Q2 during the experiment.
A schematic diagram of the measurement setup is shown in Fig. 5(a). To realize two-qubit $X Y$ gates and single-qubit phase gates $(Z)$, controlled voltage pulses generated by an arbitrary waveform generator (AWG) are used to tune the flux threading the SQUID loop of each qubit individually using flux bias lines [31]. The single-qubit microwave pulses $(X, Y)$ are generated using sideband modulation of an up-conversion in-phase quadrature (IQ) mixer [Fig. 5(b)] driven by a local oscillator (LO) and modulated by an AWG. The same up-conversion LO is used with a splitter (Sp) for the microwave pulses on both qubits to minimize the phase error introduced by phase drifts of microwave generators. We use a quantum-limited parametric amplifier (PA) to amplify readout microwave (MW) pulses at the output of R1 [Fig. 5(c)]. Here, the Josephsonjunction-based amplifier in the form of a Josephson parametric dimer (JPD) [56] is pumped by a strong pump drive through a directional coupler (D). To cancel the pump leakage, a phase-controlled $(\phi)$ and amplitude-controlled (A) microwave cancelation tone is coupled to the other port of the directional coupler (D). Three circulators (C1-C3) are used to isolate the sample from the pump tone. A circulator (C4) at base temperature followed by a cavity bandpass filter (BP) and another circulator (C5) at the still stage are used to isolate the sample and JPD from highertemperature noise. The transmitted signal is further amplified by a high-electron-mobility transistor (HEMT) at the $4.2 \mathrm{~K}$ stage and a chain of ultralow-noise (ULN) and lownoise (LN) amplifiers at room temperature, as shown in Fig. 5(d). The amplified readout pulse is down-converted from radio frequency (RF) to an intermediate frequency

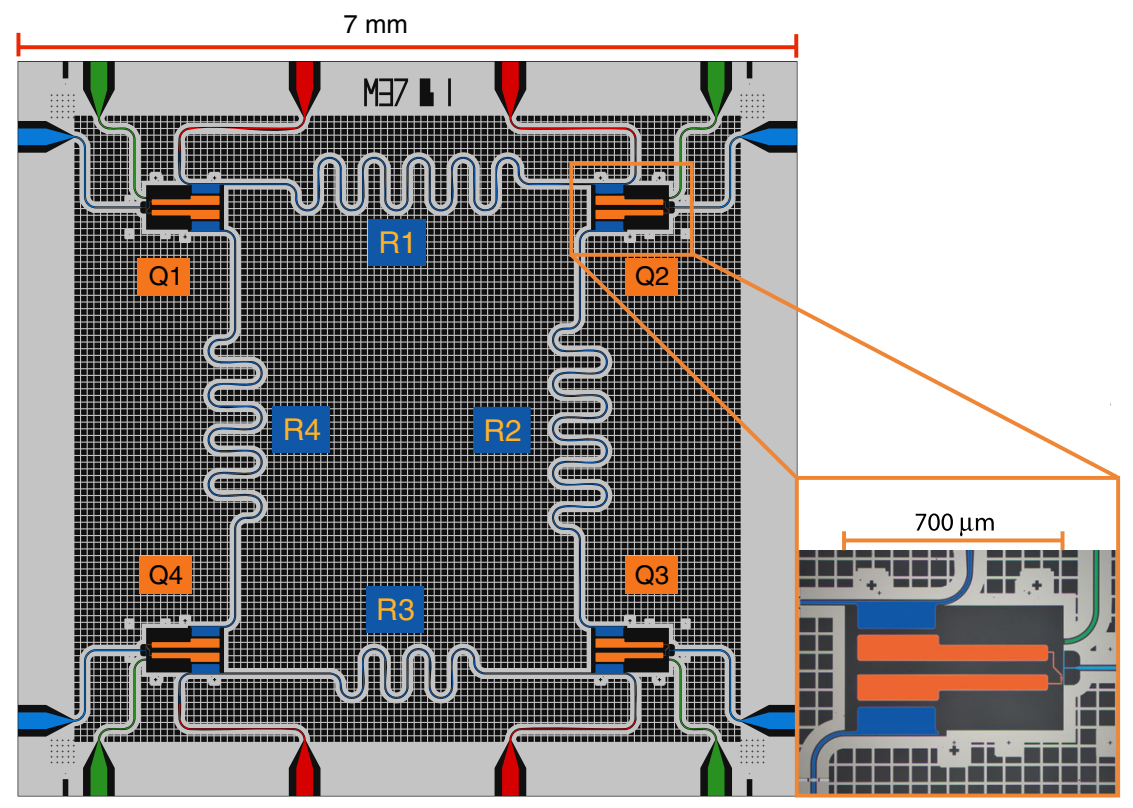

FIG. 4. Chip design and false-colored optical image of a superconducting qubit (inset). The chip comprises four superconducting qubits Q1-Q4 (orange) made of aluminium and four niobium coplanar waveguide resonators R1-R4 (deep blue) coupled to input and output ports (red). The qubits have individual microwave drive lines (green) and flux bias lines (blue). 


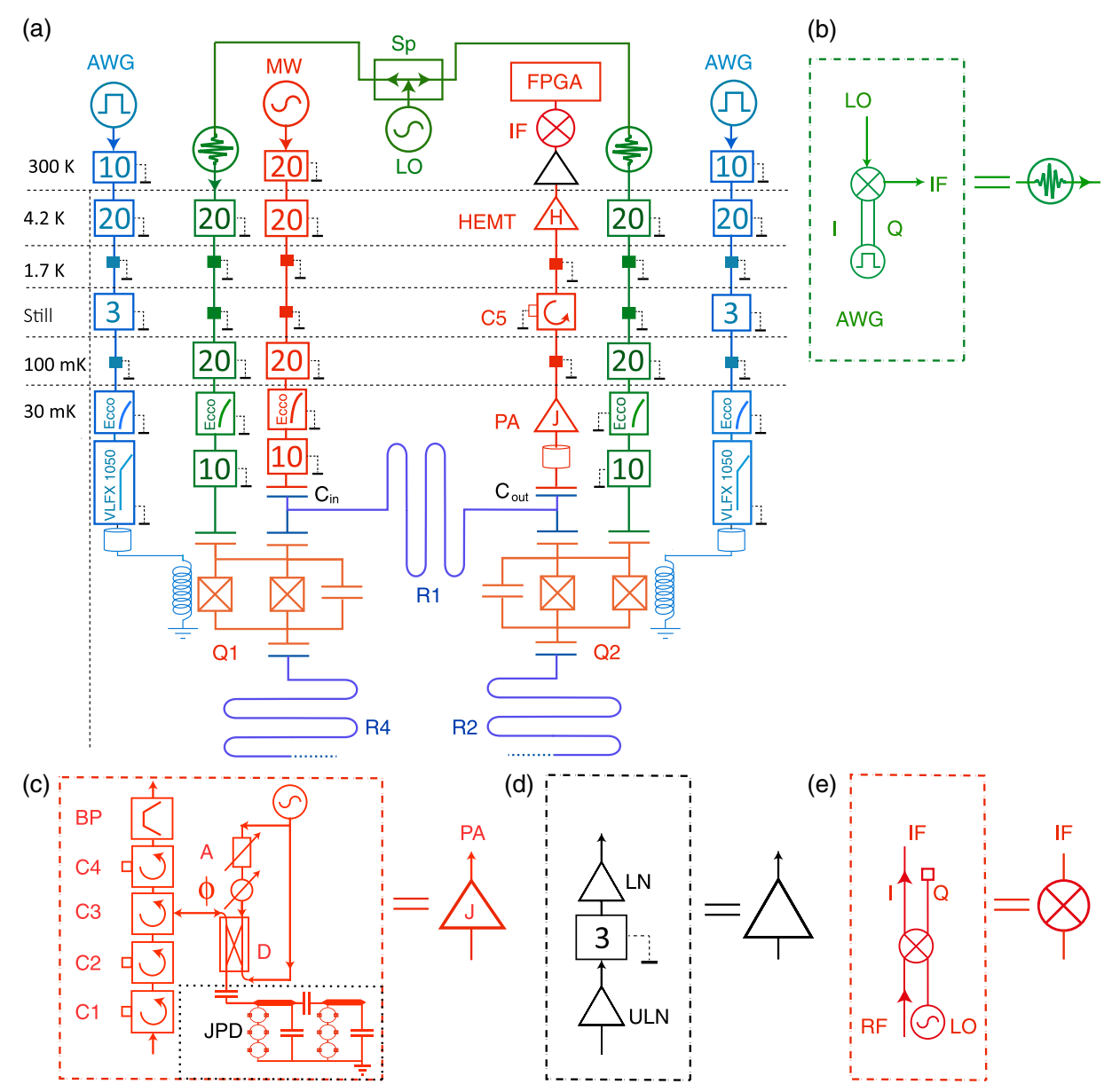

FIG. 5. (a) Schematic of the experimental setup with complete wiring of electronic components inside and outside of the dilution refrigerator with the same color code as in Fig. 4. (b) Up-conversion circuit for generating controlled microwave pulses. (c) Quantumlimited parametric amplifier circuit to amplify readout pulses at base temperature. (d) Amplifiers used at room temperature just before down-conversion of the signal. (e) Down-conversion circuit (see text for details).

(IF) of $25 \mathrm{MHz}$ using an IQ mixer [Fig. 5(e)] and digitally processed by field-programmable gate array (FPGA) logic for real-time data analysis.

\section{APPENDIX B: IMPLEMENTATION OF THE $X Y$ GATE}

The interaction between two qubits with degenerate transition frequencies dispersively coupled to the same coplanar waveguide $(\mathrm{CPW})$ resonator is described by the exchange coupling [57] $J\left(\sigma_{1}^{+} \sigma_{2}^{-}+\sigma_{1}^{-} \sigma_{2}^{+}\right)$, which can also be written in terms of Pauli operators as $\frac{J}{2}\left(\sigma_{1}^{x} \sigma_{2}^{x}+\sigma_{1}^{y} \sigma_{2}^{y}\right)$. We activate this interaction by tuning the transition frequency of qubit Q1 into resonance with qubit Q2 with a flux pulse (Fig. 6) for an interaction time $\tau$ which we varied from 0 to $60 \mathrm{~ns}$. At the frequency of qubit Q2, we obtain a coupling strength $J=-40.4 \mathrm{MHz}$ from a fit to the spectroscopically measured avoided crossing. To compensate overshoots of the flux pulse due to the limited bandwidth of the flux line channel, we use an inverted linear filter based on room-temperature response measurements of the flux line channel and in situ Ramsey measurements of the residual detuning of qubit Q1 in the time interval from 0 to $2 \mu$ s after the flux pulse.

Since the outcome of the $X Y$ gate depends strongly on the relative phase of the two-qubit input state, we use the same LO signal for the up-conversion of the single-qubit pulses acting on both qubits Q1 and Q2 [green lines in Fig. 5(a)]. Then the initial relative phase between the qubits is defined solely by the pulse sequence generated by the AWG and the cable lengths. In addition, we choose the shape of the flux pulse that realizes the $X Y$ gate such that the dynamic phase acquired by qubit Q1 during the idle time and the rising edge of the flux pulse cancels any unwanted relative phase offset of the initial state. We satisfy this condition by tuning the frequency of Q1 to an intermediate level (buffer) for a fixed time of $16 \mathrm{~ns}$ before and after the $X Y$ gate (Fig. 6). A suitable buffer level is found by performing Ramsey-type experiments with a single $X Y$ gate while sweeping the buffer amplitudes. This calibration procedure is carried out for each interaction length of the $X Y$ gate. The second buffer at the falling edge 


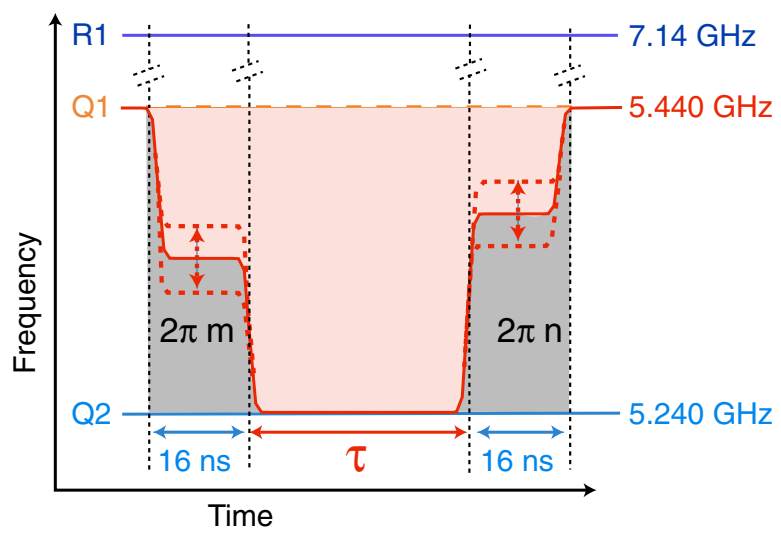

FIG. 6. Implementation of the $X Y$ gate. The transition frequency of qubit Q1 (red) is tuned into resonance with qubit Q2 (blue) for an interaction time $\tau$ using a fast flux pulse. Before and after the flux pulse, a 16-ns-long buffer is added at an intermediate level to cancel the dynamic phase accumulated by qubit Q1 relative to Q2 (gray area) during the evolution (see text). of the flux pulse is used to ascertain that the relative phase between the qubits after tuning qubit Q1 back to its original position is the same as the initial relative phase.

\section{APPENDIX C: PULSE SCHEME}

The quantum protocols for the digital quantum simulation of Heisenberg [Fig. 7(a)] and Ising spin [Fig. 7(b)] models are realized by sequences of microwave and flux pulses applied on qubit Q1 (red curves in Fig. 7) and qubit Q2 (blue curves in Fig. 7). The single-qubit rotations are implemented by 24-ns-long Gaussian-shaped resonant DRAG [58,59] microwave pulses and the $X Y$ gates are implemented using fast flux pulses. To avoid the effect of residual transient response of the flux pulse, we add a $40 \mathrm{~ns}+\Delta \tau$ waiting time after each flux pulse, with $\Delta \tau$ being an adjustable idle time. We choose $\Delta \tau$ such that the time difference between two applications of the $X Y$ interaction is commensurate with the relative phase oscillation period of $5 \mathrm{~ns}$, equal to the inverse (a)

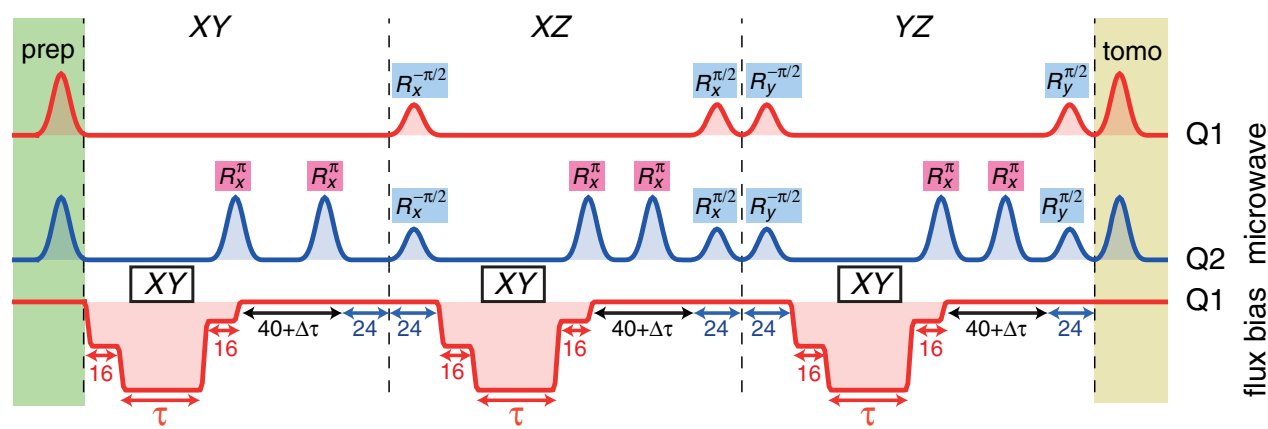

(b)

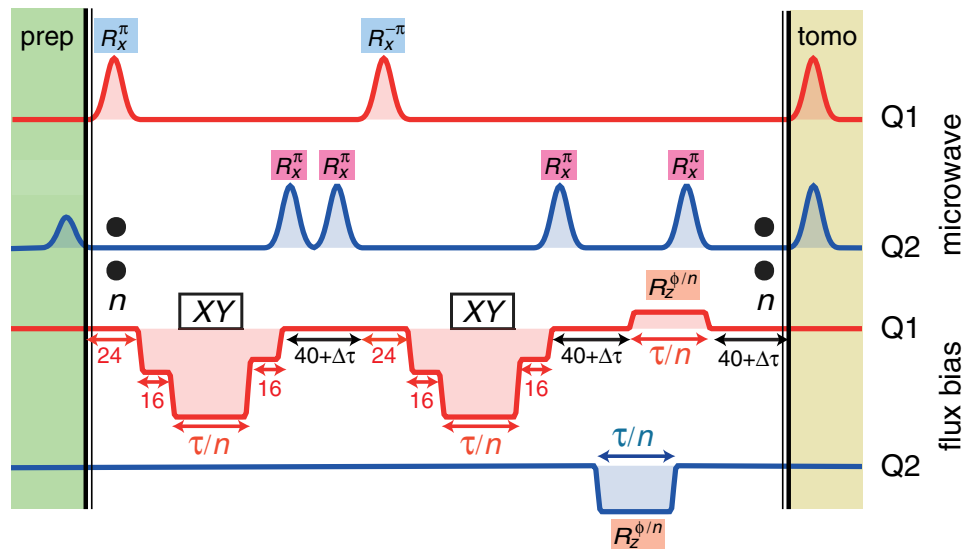

FIG. 7. Pulse sequences that are applied on qubit Q1 (red) and qubit Q2 (blue) to implement the (a) Heisenberg and (b) Ising spin models. The Gaussian-shaped microwave pulses, which are based on the derivative removal by adiabatic gate (DRAG) method, are applied to the charge lines of the respective qubits to implement single-qubit rotations $R_{x, y}^{\phi}$ about the $x$ or $y$ axis of the Bloch vector by an angle $\phi$. Each sequence starts with the preparation of an initial state (green boxes) and ends with microwave pulses for basis rotations to perform state tomography (yellow boxes). The microwave pulses marked with magenta boxes are used for refocusing. The black vertical bars with the two dots in (b) indicate that the enclosed pulse sequence is repeated $n$ times. The $X Y$ gates are realized by applying flux pulses to the flux line of qubit Q1 for a time $\tau / n$. The phase gates $R_{z}^{\phi / n}$ are implemented by detuning the transition frequency of each qubit from their idle frequencies applying flux pulses for a time $\tau / n$. The numbers stated below the pulses on qubit Q1 represent time scales in nanoseconds. 
frequency detuning $1 / 200 \mathrm{MHz}$. With these measures, we ensure that the gate can be used in a modular fashion, i.e., that a single calibration of the gate suffices for all gate realizations within the algorithm. The single-qubit phase gates are implemented by detuning the idle frequencies of each qubit with a square flux pulse. In the idle state, we observe a state-dependent qubit transition frequency shift of $940 \mathrm{kHz}$ due to the residual $\sigma_{1}^{z} \sigma_{2}^{z}$ interaction. To decouple this undesired effect, we use a standard refocusing technique [60] implemented by two consecutive $\pi$ pulses on qubit Q2 (magenta boxes in Fig. 7). In the end of each pulse sequence, we perform dispersive joint two-qubit state tomography [61] by single-qubit basis transformations followed by a pulsed microwave transmission measurement through resonator R1.

\section{APPENDIX D: PROCESS TOMOGRAPHY}

We perform standard two-qubit process tomography $[62,63]$ of the $X Y$ gate and of the simulated isotropic Heisenberg (XYZ) model for a varying interaction time $\tau$. Figure 8 shows the process $\chi$ matrices characterizing the $X Y$ gate for a quantum phase angle $\pi / 2$ [Fig. 8(a)] and $\pi$ [Fig. 8(b)] corresponding to a $\sqrt{\text { iSWAP }}$ gate $[32,64]$ and an iSWAP gate $[65,66]$ with process fidelities of $97.8 \%$ and $95.3 \%$, respectively. Heisenberg interaction with a quantum phase angle $\pi / 2$ leads to a SWAP gate [Fig. 9(a)] with a process fidelity of $86.1 \%$. While the SWAP gate belongs to the two-qubit Clifford group, there is no natural interaction in standard circuit QED architecture to directly implement the SWAP gate $[67,68]$. For a phase angle $\pi$, the Heisenberg

(a), $X Y(2|J| \tau=\pi / 2)$
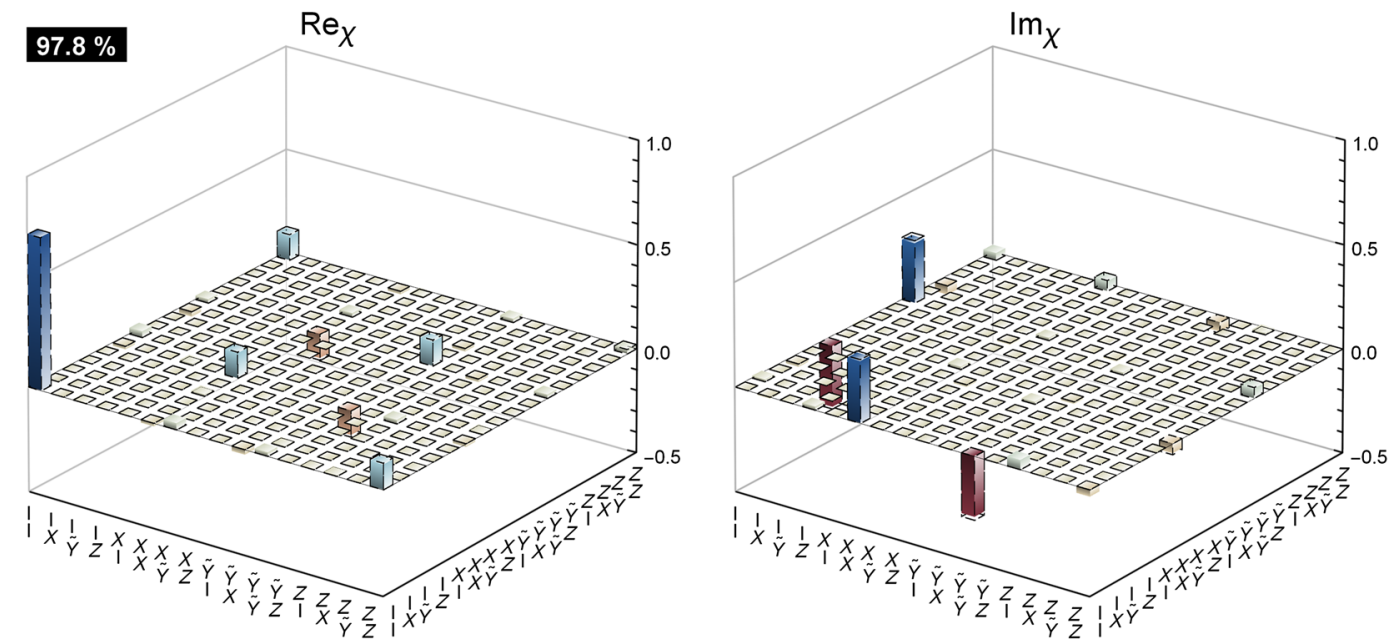

(b),$X Y(2|J| \tau=\pi)$
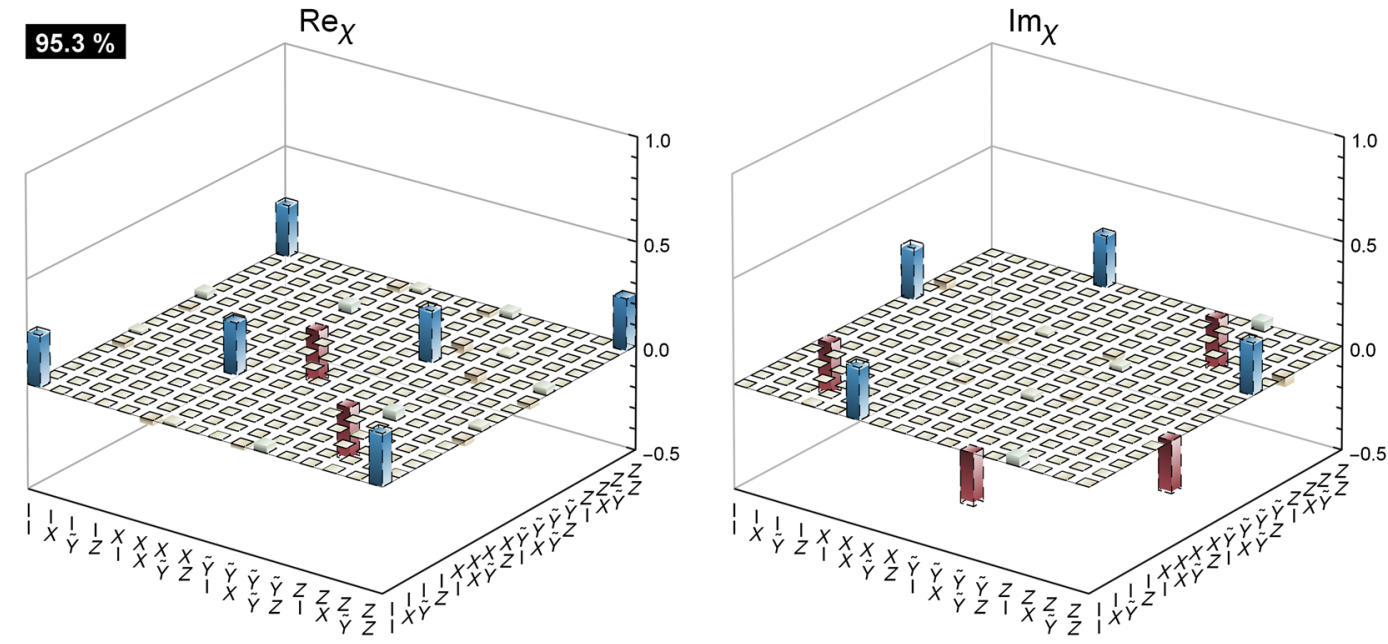

FIG. 8. (a) Measured real and imaginary parts of the $X Y$ process $\chi$ matrix $(\operatorname{Re} \chi, \operatorname{Im} \chi)$, in the basis $\left\{I=\right.$ identity, $X=\sigma_{x}$, $\left.\tilde{Y}=-i \sigma_{y}, Z=\sigma_{z}\right\}$, describing the mapping from any initial state to the final state for a quantum phase angle of $2|J| \tau=\pi / 2$. The dashed wire frames represent the theoretically optimal matrix elements and the colored bars represent measured positive (blue) and negative (red) matrix elements. The fidelity of the experimentally observed process with respect to the ideal process is indicated in the black boxes. (b) As in (a) for a phase angle $\pi$. 



(b),$X Y Z(2|J| \tau=\pi)$
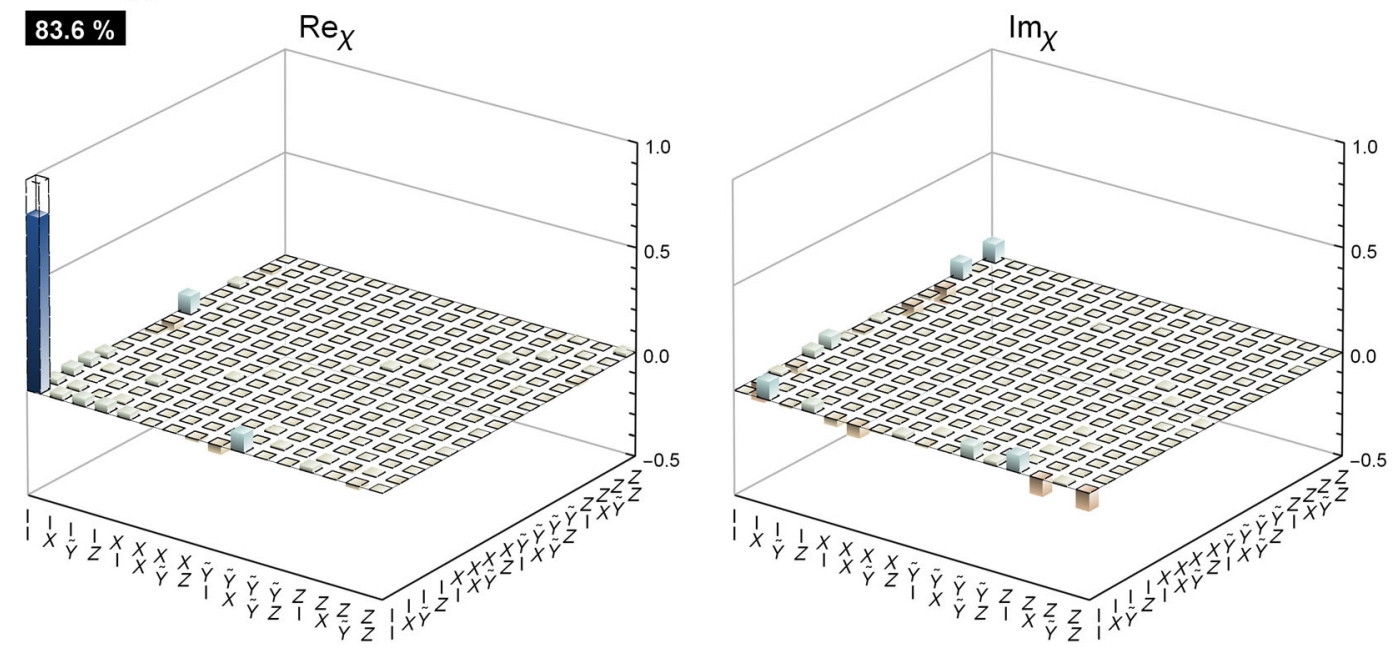

FIG. 9. (a) Measured real and imaginary parts of the Heisenberg $(X Y Z)$ process $\chi$ matrix ( $\operatorname{Re} \chi$, $\operatorname{Im} \chi)$, in the basis $\left\{I=\right.$ identity, $\left.X=\sigma_{x}, \tilde{Y}=-i \sigma_{y}, Z=\sigma_{z}\right\}$, describing the mapping from any initial state to the final state for a quantum phase angle of $2|J| \tau=\pi / 2$. The dashed wire frames represent the theoretically optimal matrix elements and the colored bars represent measured positive (blue) and negative (red) matrix elements. The fidelity of the experimentally observed process with respect to the ideal process is indicated in the black boxes. (b) As in (a) for a phase angle $\pi$.

interaction is an identity gate [Fig. 9(b)] with a process fidelity of $83.6 \%$.

\section{APPENDIX E: ERROR CONTRIBUTIONS}

The single-qubit gate fidelities measured by randomized benchmarking [69-71] amount to $99.7 \%$. The dominant contribution to the loss in fidelity originates from the twoqubit $X Y$ gates for which a process fidelity $\mathcal{F}_{p, X Y}=95.7 \%$ is obtained from process tomography averaging over all quantum phase angles. This indicates that the errors in the implementation of the $X Y$ gate limit the fidelity of the final state of the quantum simulation. To confirm this, we calculate the expected process fidelity for the Heisenberg and Ising protocol from the observed $X Y$ gate fidelity by assuming independent gate errors in all three steps. For the
Heisenberg $(X Y Z)$ model simulation neglecting the small single-qubit gate errors, we expect a mean process fidelity $\mathcal{F}_{p, X Y Z} \approx 1-3\left(1-\mathcal{F}_{p, X Y}\right)=87.1 \%$, which is close to the observed value of $86.3 \%$. For the Ising model simulation, we expect a process fidelity of $\mathcal{F}_{p \text {,Ising }} \approx 1-2 n\left(1-\mathcal{F}_{p, X Y}\right)$. From the relation $\mathcal{F}_{s}=\left(d \mathcal{F}_{p}+1\right) /(d+1)$ between state $\left(\mathcal{F}_{s}\right)$ and process fidelity $\left(\mathcal{F}_{p}\right)$, we obtain the expected mean state fidelities of $\{93.1,86.2,79.4,72.5,65.6\} \%$ for $n=1-5$ Trotter steps, which compare well to the measured state fidelites $\{91.7,88.3,82.2,73.0,60.7\} \%$.

To estimate the dominant source of systematic errors, we consider a model that includes relaxation $\left(T_{1}\right)$ and dephasing $\left(T_{2}\right)$ and state-dependent phase errors described by an effective $\tilde{J}_{z} \sigma_{1}^{z} \sigma_{2}^{z}$ term with interaction strength $\tilde{J}_{z}$. In addition, we include an extra offset in the single-qubit 
phase gate acting on qubit Q2 from cross talk of the flux pulses acting on qubit Q1 in each Trotter step. By fitting the final state predicted by this model to the observed states, we estimate an unwanted interaction angle $\tilde{J}_{z} \tau_{z}$ of approximately $2.3^{\circ}$ and a constant phase offset of $4.6^{\circ}$.

[1] R. P. Feynman, Simulating physics with computers, Int. J. Theor. Phys. 21, 467 (1982).

[2] S. Lloyd, Universal quantum simulators, Science 273, 1073 (1996).

[3] L. Balents, Spin liquids in frustrated magnets, Nature (London) 464, 199 (2010).

[4] S. Sachdev, Quantum Phase Transitions (Cambridge University Press, Cambridge, England, 1999).

[5] P. B. Lanyon, J. D. Whitfield, G. G. Gillett, M. E. Goggin, M. P. Almeida, I. Kassal, J. D. Biamonte, M. Mohseni, B. J. Powell, M. Barbieri, A. Aspuru-Guzik, and A. G. White, Towards quantum chemistry on a quantum computer, Nat. Chem. 2, 106 (2010).

[6] J. I. Cirac, P. Maraner, and J. K. Pachos, Cold Atom Simulation of Interacting Relativistic Quantum Field Theories, Phys. Rev. Lett. 105, 190403 (2010).

[7] A. Bermudez, L. Mazza, M. Rizzi, N. Goldman, M. Lewenstein, and M. A. Martin-Delgado, Wilson Fermions and Axion Electrodynamics in Optical Lattices, Phys. Rev. Lett. 105, 190404 (2010).

[8] P. W. Anderson, The Theory of Superconductivity in the High- $T_{c}$ Cuprate Superconductors (Princeton University Press, Princeton, NJ, 1997).

[9] C. Gogolin, M.P. Müller, and J. Eisert, Absence of Thermalization in Nonintegrable Systems, Phys. Rev. Lett. 106, 040401 (2011).

[10] A. Polkovnikov, K. Sengupta, A. Silva, and M. Vengalattore, Nonequilibrium dynamics of closed interacting quantum systems, Rev. Mod. Phys. 83, 863 (2011).

[11] R. Blatt and C. Roos, Quantum simulations with trapped ions, Nat. Phys. 8, 277 (2012).

[12] I. Bloch, J. Dalibard, and S. Nascimbène, Quantum simulations with ultracold quantum gases, Nat. Phys. 8, 267 (2012).

[13] A. Aspuru-Guzik and P. Walther, Photonic quantum simulators, Nat. Phys. 8, 285 (2012).

[14] A. Friedenauer, H. Schmitz, J. T. Glueckert, D. Porras, and T. Schaetz, Simulating a quantum magnet with trapped ions, Nat. Phys. 4, 757 (2008).

[15] B. P. Lanyon, C. Hempel, D. Nigg, M. Müller, R. Gerritsma, F. Zähringer, P. Schindler, J. T. Barreiro, M. Rambach, G. Kirchmair, M. Hennrich, P. Zoller, R. Blatt, and C. F. Roos, Universal digital quantum simulation with trapped ions, Science 334, 57 (2011).

[16] U. Las Heras, A. Mezzacapo, L. Lamata, S. Filipp, A. Wallraff, and E. Solano, Digital Quantum Simulation of Spin Systems in Superconducting Circuits, Phys. Rev. Lett. 112, 200501 (2014).

[17] M. Greiner, O. Mandel, T. Esslinger, T. W. Hansch, and I. Bloch, Quantum phase transition from a superfluid to a mott insulator in a gas of ultracold atoms, Nature (London) 415, 39 (2002).
[18] R. Gerritsma, G. Kirchmair, F. Zahringer, E. Solano, R. Blatt, and C.F. Roos, Quantum simulation of the Dirac equation, Nature (London) 463, 68 (2010).

[19] A. A. Houck, H. E. Tureci, and J. Koch, On-chip quantum simulation with superconducting circuits, Nat. Phys. 8, 292 (2012).

[20] P. Roushan et al., Observation of topological transitions in interacting quantum circuits, Nature (London) 515, 241 (2014).

[21] M. D. Schroer, M. H. Kolodrubetz, W. F. Kindel, M. Sandberg, J. Gao, M. R. Vissers, D. P. Pappas, A. Polkovnikov, and K. W. Lehnert, Measuring a Topological Transition in an Artificial Spin-1/2 System, Phys. Rev. Lett. 113, 050402 (2014).

[22] R. Barends et al., Digital Quantum Simulation of Fermionic Models with a Superconducting Circuit, arXiv:1501.07703.

[23] M. Suzuki, Fractal decomposition of exponential operators with applications to many-body theories and Monte Carlo simulations, Phys. Lett. A 146, 319 (1990).

[24] N. Wiebe, D. W. Berry, P. Høyer, and B. C. Sanders, Simulating quantum dynamics on a quantum computer, J. Phys. A 44, 445308 (2011).

[25] A. M. Childs and N. Wiebe, Hamiltonian simulation using linear combinations of unitary operations, Quantum Inf. Comput. 12, 901 (2012).

[26] A. Wallraff, D. I. Schuster, A. Blais, L. Frunzio, R.-S. Huang, J. Majer, S. Kumar, S. M. Girvin, and R. J. Schoelkopf, Strong coupling of a single photon to a superconducting qubit using circuit quantum electrodynamics, Nature (London) 431, 162 (2004).

[27] J. Koch, T. M. Yu, J. Gambetta, A. A. Houck, D. I. Schuster, J. Majer, A. Blais, M. H. Devoret, S. M. Girvin, and R. J. Schoelkopf, Charge-insensitive qubit design derived from the cooper pair box, Phys. Rev. A 76, 042319 (2007).

[28] J. Majer, J. M. Chow, J. M. Gambetta, J. Koch, B. R. Johnson, J. A. Schreier, L. Frunzio, D. I. Schuster, A. A. Houck, A. Wallraff, A. Blais, M. H. Devoret, S. M. Girvin, and R. J. Schoelkopf, Coupling superconducting qubits via a cavity bus, Nature (London) 449, 443 (2007).

[29] R. Bianchetti, S. Filipp, M. Baur, J. M. Fink, M. Göppl, P. J. Leek, L. Steffen, A. Blais, and A. Wallraff, Dynamics of dispersive single-qubit readout in circuit quantum electrodynamics, Phys. Rev. A 80, 043840 (2009).

[30] S. Filipp, M. Göppl, J. M. Fink, M. Baur, R. Bianchetti, L. Steffen, and A. Wallraff, Multimode mediated qubit-qubit coupling and dark-state symmetries in circuit quantum electrodynamics, Phys. Rev. A 83, 063827 (2011).

[31] L. DiCarlo, J. M. Chow, J. M. Gambetta, L. S. Bishop, B. R. Johnson, D. I. Schuster, J. Majer, A. Blais, L. Frunzio, S. M. Girvin, and R. J. Schoelkopf, Demonstration of two-qubit algorithms with a superconducting quantum processor, Nature (London) 460, 240 (2009).

[32] A. Dewes, F. R. Ong, V. Schmitt, R. Lauro, N. Boulant, P. Bertet, D. Vion, and D. Esteve, Characterization of a Two-Transmon Processor with Individual Single-Shot Qubit Readout, Phys. Rev. Lett. 108, 057002 (2012).

[33] G. Vidal and R.F. Werner, Computable measure of entanglement, Phys. Rev. A 65, 032314 (2002).

[34] D. W. Berry, G. Ahokas, R. Cleve, and B. C. Sanders, Efficient quantum algorithms for simulating sparse Hamiltonians, Commun. Math. Phys. 270, 359 (2007). 
[35] D. J. Egger and F. K. Wilhelm, Adaptive Hybrid Optimal Quantum Control for Imprecisely Characterized Systems, Phys. Rev. Lett. 112, 240503 (2014).

[36] E. Jeffrey, D. Sank, J. Y. Mutus, T. C. White, J. Kelly, R. Barends, Y. Chen, Z. Chen, B. Chiaro, A. Dunsworth, A. Megrant, P. J. J. O’Malley, C. Neill, P. Roushan, A. Vainsencher, J. Wenner, A. N. Cleland, and J. M. Martinis, Fast Accurate State Measurement with Superconducting Qubits, Phys. Rev. Lett. 112, 190504 (2014).

[37] R. Schutjens, F. A. Dagga, D. J. Egger, and F. K. Wilhelm, Single-qubit gates in frequency-crowded transmon systems, Phys. Rev. A 88, 052330 (2013).

[38] J. M. Hornibrook, J. I. Colless, I. D. Conway Lamb, S. J. Pauka, H. Lu, A. C. Gossard, J. D. Watson, G. C. Gardner, S. Fallahi, M. J. Manfra, and D. J. Reilly, Cryogenic Control Architecture for Large-Scale Quantum Computing, Phys. Rev. Applied 3, 024010 (2015).

[39] J. M. Chow, L. DiCarlo, J. M. Gambetta, F. Motzoi, L. Frunzio, S. M. Girvin, and R. J. Schoelkopf, Optimized driving of superconducting artificial atoms for improved single-qubit gates, Phys. Rev. A 82, 040305 (2010).

[40] D. Ristè, J. G. van Leeuwen, H.-S. Ku, K. W. Lehnert, and L. DiCarlo, Initialization by Measurement of a Superconducting Quantum Bit Circuit, Phys. Rev. Lett. 109, 050507 (2012).

[41] D. Ristè, C. C. Bultink, K. W. Lehnert, and L. DiCarlo, Feedback Control of a Solid-State Qubit Using HighFidelity Projective Measurement, Phys. Rev. Lett. 109, 240502 (2012).

[42] B. Yurke and E. Buks, Performance of cavity-parametric amplifiers, employing kerr nonlinearites, in the presence of two-photon loss, J. Lightwave Technol. 24, 5054 (2006).

[43] M. A. Castellanos-Beltran and K. W. Lehnert, Widely tunable parametric amplifier based on a superconducting quantum interference device array resonator, Appl. Phys. Lett. 91, 083509 (2007).

[44] R. Islam, C. Senko, W. C. Campbell, S. Korenblit, J. Smith, A. Lee, E. E. Edwards, C.-C. J. Wang, J. K. Freericks, and C. Monroe, Emergence and frustration of magnetism with variable-range interactions in a quantum simulator, Science 340, 583 (2013).

[45] X.-S. Ma, B. Dakic, W. Naylor, A. Zeilinger, and P. Walther, Quantum simulation of the wavefunction to probe frustrated Heisenberg spin systems, Nat. Phys. 7, 399 (2011).

[46] P. Richerme, Z.-X. Gong, A. Lee, C. Senko, J. Smith, M. Foss-Feig, S. Michalakis, A. V. Gorshkov, and C. Monroe, Non-local propagation of correlations in quantum systems with long-range interactions, Nature (London) 511, 198 (2014).

[47] P. Jurcevic, B. P. Lanyon, P. Hauke, C. Hempel, P. Zoller, R. Blatt, and C.F. Roos, Quasiparticle engineering and entanglement propagation in a quantum many-body system, Nature (London) 511, 202 (2014).

[48] T. Fukuhara, P. Schauß, M. Endres, S. Hild, M. Cheneau, I. Bloch, and C. Gross, Microscopic observation of magnon bound states and their dynamics, Nature (London) 502, 76 (2013).

[49] A. Chiesa, P. Santini, D. Gerace, J. Raftery, A. A. Houck, and S. Carretta, Digital Quantum Simulators in a
Scalable Architecture of Hybrid Spin-Photon Qubits, arXiv:1504.05667.

[50] H. Lu, C. Liu, D.-S. Wang, L.-K. Chen, Z.-D. Li, X.-C. Yao, L. Li, N.-L. Liu, C.-Z. Peng, B. C. Sanders, Y.-A. Chen, and J.-W. Pan, Universal Digital Photonic Single-Qubit Quantum Channel Simulator, arXiv:1505.02879.

[51] A. Mezzacapo, U. Las Heras, J. S. Pedernales, L. DiCarlo, E. Solano, and L. Lamata, Digital quantum Rabi and Dicke models in superconducting circuits, Sci. Rep. 4, 7482 (2014).

[52] L. Steffen, Y. Salathe, M. Oppliger, P. Kurpiers, M. Baur, C. Lang, C. Eichler, G. Puebla-Hellmann, A. Fedorov, and A. Wallraff, Deterministic quantum teleportation with feed-forward in a solid state system, Nature (London) 500, 319 (2013).

[53] D. Ristè, S. Poletto, M.-Z. Huang, A. Bruno, V. Vesterinen, O.-P. Saira, and L. DiCarlo, Detecting bit-flip errors in a logical qubit using stabilizer measurements, Nat. Commun. 6, 6983 (2015).

[54] J. Kelly et al., State preservation by repetitive error detection in a superconducting quantum circuit, Nature (London) 519, 66 (2015).

[55] J. M. Chow, J. M. Gambetta, E. Magesan, D. W. Abraham, A. W. Cross, B. R. Johnson, N. A. Masluk, C. A. Ryan, J. A. Smolin, S. J. Srinivasan, and M. Steffen, Implementing a strand of a scalable fault-tolerant quantum computing fabric, Nat. Commun. 5, 4015 (2014).

[56] C. Eichler, Y. Salathe, J. Mlynek, S. Schmidt, and A. Wallraff, Quantum-Limited Amplification and Entanglement in Coupled Nonlinear Resonators, Phys. Rev. Lett. 113, 110502 (2014).

[57] A. Blais, R.-S. Huang, A. Wallraff, S. M. Girvin, and R. J. Schoelkopf, Cavity quantum electrodynamics for superconducting electrical circuits: An architecture for quantum computation, Phys. Rev. A 69, 062320 (2004).

[58] F. Motzoi, J. M. Gambetta, P. Rebentrost, and F. K. Wilhelm, Simple Pulses for Elimination of Leakage in Weakly Nonlinear Qubits, Phys. Rev. Lett. 103, 110501 (2009).

[59] J. M. Gambetta, F. Motzoi, S. T. Merkel, and F. K. Wilhelm, Analytic control methods for high-fidelity unitary operations in a weakly nonlinear oscillator, Phys. Rev. A 83, 012308 (2011).

[60] L. M. K. Vandersypen and I. L. Chuang, NMR techniques for quantum control and computation, Rev. Mod. Phys. 76, 1037 (2005).

[61] S. Filipp, P. Maurer, P. J. Leek, M. Baur, R. Bianchetti, J. M. Fink, M. Göppl, L. Steffen, J. M. Gambetta, A. Blais, and A. Wallraff, Two-Qubit State Tomography Using a Joint Dispersive Readout, Phys. Rev. Lett. 102, 200402 (2009).

[62] J. F. Poyatos, J. I. Cirac, and P. Zoller, Complete Characterization of a Quantum Process: The Two-Bit Quantum Gate, Phys. Rev. Lett. 78, 390 (1997).

[63] I. L. Chuang and M. A. Nielsen, Prescription for experimental determination of the dynamics of a quantum black box, J. Mod. Opt. 44, 2455 (1997).

[64] A. G. Kofman and A. N. Korotkov, Two-qubit decoherence mechanisms revealed via quantum process tomography, Phys. Rev. A 80, 042103 (2009). 
[65] N. Schuch and J. Siewert, Natural two-qubit gate for quantum computation using the $X Y$ interaction, Phys. Rev. A 67, 032301 (2003).

[66] M. Neeley, R. C. Bialczak, M. Lenander, E. Lucero, M. Mariantoni, A. D. O'Connell, D. Sank, H. Wang, M. Weides, J. Wenner, Y. Yin, T. Yamamoto, A. N. Cleland, and J. M. Martinis, Generation of three-qubit entangled states using superconducting phase qubits, Nature (London) 467, 570 (2010).

[67] T. Wu and L. Ye, Implementing two-qubit SWAP gate with SQUID qubits in a microwave cavity via adiabatic passage evolution, Int. J. Theor. Phys. 51, 1076 (2012).

[68] A. D. Córcoles, J. M. Gambetta, J. M. Chow, J. A. Smolin, M. Ware, J. Strand, B. L. T. Plourde, and M. Steffen,
Process verification of two-qubit quantum gates by randomized benchmarking, Phys. Rev. A 87, 030301 (2013).

[69] E. Knill, D. Leibfried, R. Reichle, J. Britton, R. B. Blakestad, J. D. Jost, C. Langer, R. Ozeri, S. Seidelin, and D. J. Wineland, Randomized benchmarking of quantum gates, Phys. Rev. A 77, 012307 (2008).

[70] J. M. Chow, J. M. Gambetta, L. Tornberg, J. Koch, L. S. Bishop, A. A. Houck, B. R. Johnson, L. Frunzio, S. M. Girvin, and R. J. Schoelkopf, Randomized Benchmarking and Process Tomography for Gate Errors in a Solid-State Qubit, Phys. Rev. Lett. 102, 090502 (2009).

[71] J. Kelly et al., Optimal Quantum Control Using Randomized Benchmarking, Phys. Rev. Lett. 112, 240504 (2014). 